\title{
Heparin priming of EUS-FNA needles does not adversely affect tissue cytology or immunohistochemical staining $\square$
}

\section{(ㄷ)(1) $\odot$}

\author{
Authors \\ David L. Diehl, Shaffer R. S. Mok, Harshit S. Khara, Amitpal S. Johal, H. Lester Kirchner, Fan Lin
}

Institution

Geisinger Medical Center, Department of Gastroenterology and Hepatology, Division of Interventional Endoscopy, Danville, Pennsylvania, United States

submitted 16.6.2017

accepted after revision 31.8 .2017

Bibliography

DOI https://doi.org/10.1055/s-0043-121880 |

Endoscopy International Open 2018; 06: E356-E362

(c) Georg Thieme Verlag KG Stuttgart · New York

ISSN 2364-3722

Corresponding author

Shaffer R. S. Mok, MD, MBS, Geisinger Medical Center, Department of Gastroenterology and Hepatology, Division of Interventional Endoscopy, 100 North Academy Ave, Danville, PA, 17822

Fax: +1-570-271-6852

mok.shaffer@gmail.com

\section{ABSTRACT}

Background and study aims Endoscopic ultrasound (EUS)-guided fine-needle aspiration (FNA) or biopsy (FNB) is an indispensable diagnostic tool. Improvements in needling technique have led to increasing tissue yields. Blood clogging of the needle can cause difficulties with specimen handling and stylet passage, which improves when the needle is primed with heparin before use. However, the effect of heparin on cytology, histology or immunochemistry (IHC) of FNA and FNB specimens is unknown. The goal of the study was to evaluate heparin priming on cytologic/histologic appearance, IHC staining, ease of stylet passage, and specimen bloodiness.

Patients and methods This was a retrospective study of patients undergoing EUS-FNA/FNB. Needle sizes were 25 gauge $(\mathrm{g}), 22 \mathrm{~g}$, and $19 \mathrm{~g}$. Heparin priming of the needle was done and the stylet replaced ("dry heparin") or suction attached without replacing the stylet ("wet heparin"). Smears and cellblocks were examined by pathologists, and IHC staining were done as needed. Specimen bloodiness was compared with matched controls.

Results Adequate tissue yields were obtained in all samples (37 heparin, 36 no heparin). Heparin priming did not exhibit negative effects on cytologic or histologic interpretation of the specimens, nor IHC. There was no difference in cellblock bloodiness between the heparin primed needle specimens and the non-heparin control group.

Conclusions Heparin priming of EUS-FNA or FNB needles does not negatively affect cytologic or histologic interpretation, nor interfere with IHC. In addition, heparin priming does not increase specimen bloodiness. When the "wet suction" technique is used for EUS-FNA, heparin priming can be used instead of saline priming of the EUS needle.

\section{Introduction}

Endoscopic ultrasound (EUS) with fine-needle aspiration (FNA) has become an indispensable resource for obtaining a pathological diagnosis in a variety of clinical conditions. Adequate tissue for diagnosis can be obtained with high sensitivity, specificity and safety [1]. Exciting new developments in needle design, and the ability to deliver a "core" specimen are likely to further advance the diagnostic ability of EUS. Similarly, investigations to determine the optimal needling technique and suction strengths have yielded new and important information [2-4].
For example, "fanning" the EUS-FNA needle within the target has become the standard technique for solid lesions [5]. Minimal or no suction, for example via the "slow pull" technique, also seems to be useful for delivering specimens with less blood and more tissue [6]. The "wet suction" technique has also shown promise in delivering higher tissue yields [7]. In this technique, the needle is flushed with saline prior to FNA with suction. In a prospective comparison to conventional FNA technique, wet suction yielded higher tissue cellularity and specimen adequacy.

The accumulation of blood with successive EUS- FNA passes can lower the quality of the specimen [8]. Additionally, blood clogging can make it increasingly difficult to insert the stylet 
into the needle lumen. Given these two negative aspects of blood contamination, it may be hypothesized that priming the needle with an anticoagulant such as heparin could resolve these issues.

The technique of flushing a needle with heparin has been evaluated for EUS-FNA in only 2 studies [9-10]. With the increasing importance of immunohistochemical (IHC) characterization of FNA tissue, it is vital to assess if heparin priming alters IHC staining of these specimens. The aims of this study were to demonstrate that use of a heparin-primed EUS-FNA needle does not disturb cytologic or histologic architecture, and that IHC evaluation of these specimens is not adversely affected.

\section{Patients and methods}

This pilot study was performed using retrospectively collected data obtained between January 2013 to September 2016 at a tertiary care center in the United States. This study received approval from our Institutional Review Board.

\section{Patients}

Patients undergoing EUS for solid lesions in or adjacent to the gastrointestinal lumen were included. Use of heparin priming of the EUS needle served as the case group, and no-heparin as the control group. To provide a non-heparin needle control group, cases were matched to a group of EUS-FNA procedures done in a period (2013-2014) before heparin priming of needles was adopted. Heparin specimens were matched to controls using similar FNA targets.

Patients were excluded if any of the following criteria were present: age under 18 years, pregnancy, allergy to heparin products, a religious reason for avoidance of porcine products, INR $>1.5$, and platelet count $<50,000$.

\section{Device selection}

Procedures were done by three experienced endosonographers who perform about 400 EUS procedures/year. Interventional endoscopy fellows took part in the procedures. Prior to EUS, all patients underwent standard pre-procedural evaluation by the participating endosonographer and an anesthesiologist. Selective pre-procedural labs were obtained when there was a possibility of abnormal hemostatic parameters (e.g. platelet count and INR for patients with liver disease). Sedation was done with monitored anesthesia care with propofol administered by a nurse anesthetist. Endosonographic examination was performed to locate the target lesions for each patient. Prior to needle puncture, color Doppler imaging was utilized to identify vessels and ensure a safe needle trajectory into the target lesion.

Both Boston Scientific (Marlborough, MA) $22 \mathrm{~g}$ and $25 \mathrm{~g}$, and $22 \mathrm{~g}$ Shark Core (Medtronic Inc, Minneapolis, MN) EUS needles were used for the solid lesions sampled in this series.

\section{Heparin EUS technique}

EUS-FNA of a variety of lesions was done using standard means with either dry or wet technique done at the discretion of the endoscopist. Definitions of both wet and dry heparin priming are described below. Standard FNA technique, including fanning, was done in all cases.

Before use, the EUS needle was primed with heparin flush (500 USP units heparin/ $5 \mathrm{~mL}$ ), $5 \mathrm{~mL}$ fill in 12-mL syringe (Medefil, Inc., Glendale Heights, IL) until drops of the flush came out of the needle tip. The stylet was reinserted ("dry heparin" technique), or the heparin was left in the needle lumen and a suction syringe attached to the needle hub ("wet heparin" technique).

Slow-pull suction was done for the dry heparin approach, and the needle primed with heparin only the first time, and not on subsequent passes. Wet heparin technique used full suction $(20 \mathrm{~mL})$. After FNA, the stylet was inserted into the needle to deliver the specimen. The stylet was then removed and the needle again primed with heparin prior to the next pass. The number of passes into the lesion was determined at the discretion of the endoscopist ( $\triangleright$ Video 1 ).

\section{Specimen collection and processing}

A small portion of the specimen was expressed from the needle onto a slide, and a cytologic smear made. One slide was air dried and stained with Diff-Quik stain, and the other sprayed with cytology fixative (Leica, Richmond, IL) for Papanicolaou stain. The remaining needle contents were placed into RPMI transport medium, and transported to the cytology receiving lab. Flow cytometry was done in selected cases as needed. Rapid on-site cytologic evaluation was done when requested by the endoscopist. IHC stains were performed using a Ventana Discovery Ultra automated staining platform (Ventana Medical Systems, Tucson, AZ) as ordered by the cytopathologist for lesion characterization. Both smears (air dried and fixed), cellblocks, and IHC stains were reviewed by the cytopathologist, who then made a pathological diagnosis.

Cellblock H\&E slides were pulled from slide files. Slides were blinded and order of microscopic reading randomized. Bloodiness of the cellblock specimens was rated ( $\triangleright$ Fig. 1 ) according

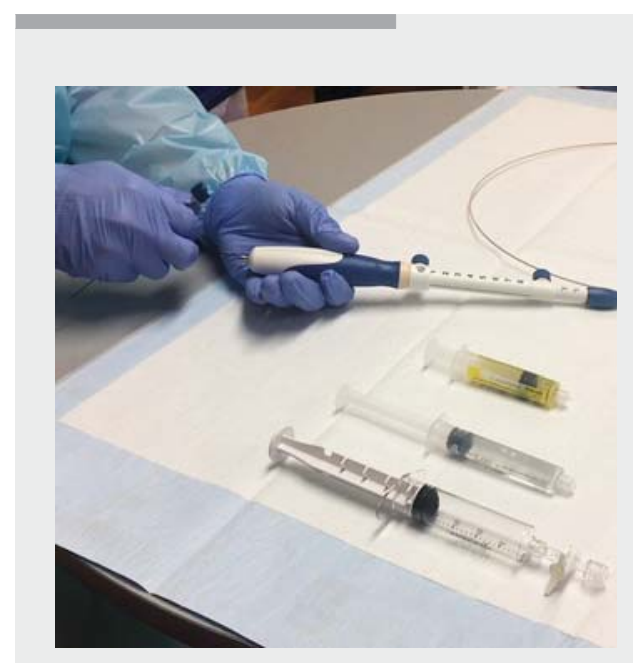

Video 1 This video depicts the stepwise process of creating dry and wet heparin using a standard FNA needle. 


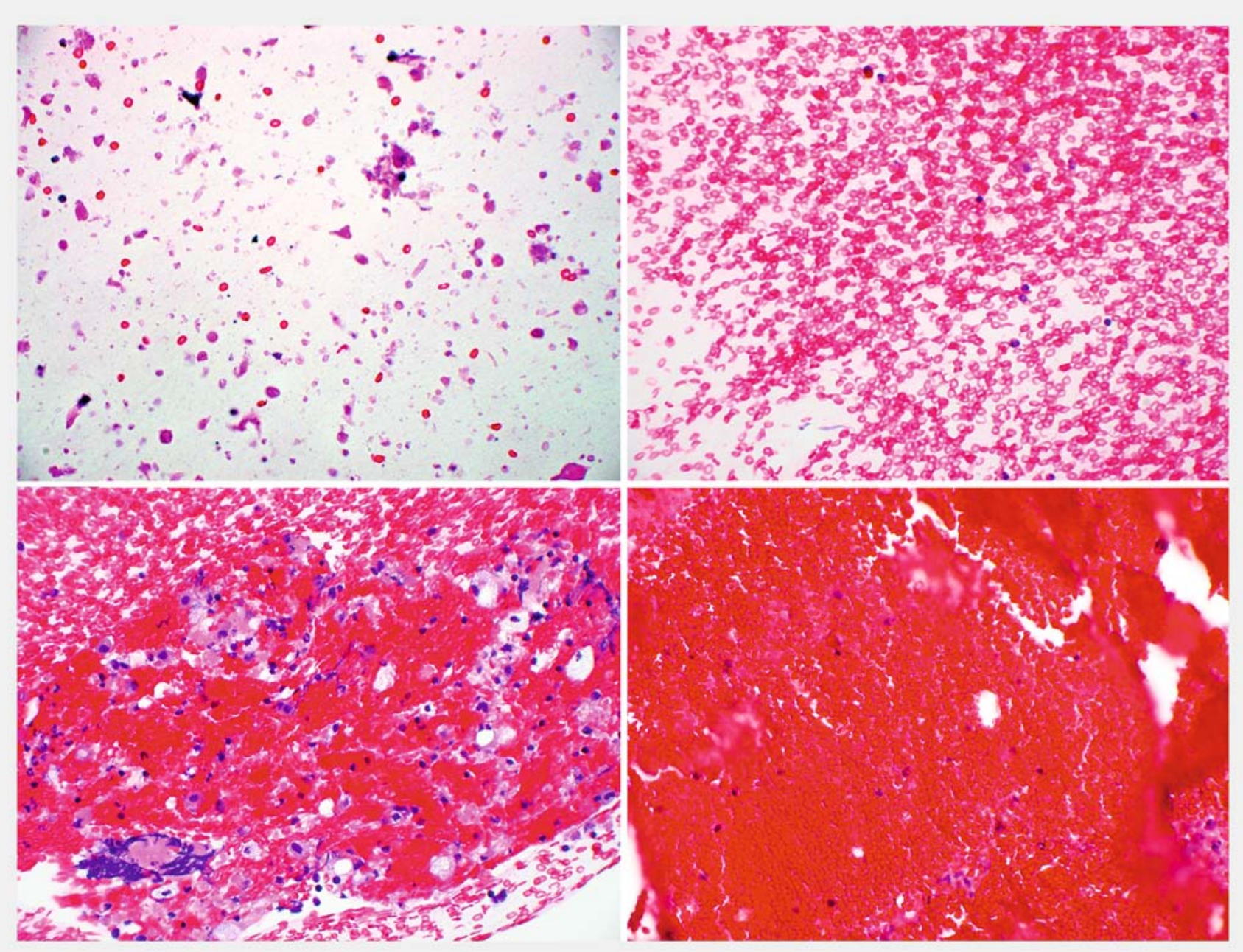

- Fig. 1 Grading scale of blood of cell block specimen. Photomicrographs show grading of the degree of blood present in a specimen. 0 (A): nearly absent of RBC; $1+(B)$ : monolayer of RBC, no cluster formation; $2+(C)$ : aggregates of RBC, <1 per high power field (HPF) (x400); $3+(D)$ : aggregates of RBC present, $>1$ per HPF $(\times 400)$

to the following scale: 0 : nearly absent of red blood cell (RBC); 1 + : monolayer of RBC, no cluster formation; $2+$ : aggregates of RBC present, < one high power field $(\times 400) ; 3+$ : aggregates of RBC present, > one high power field $(\times 400)$.

\section{Outcomes}

The primary outcome was the ability to perform IHC staining and obtain a final pathologic diagnosis. Secondary outcomes included the nature and organ of the lesion sampled, number of needle passes, final pathological diagnosis, cellblock bloodiness, use of flow cytometry, and IHC stains. All data was obtained via review of our institution's electronic medical records to evaluate procedural and pathology results. Post-procedure complications if any, were sought by telephone calls to patients 24 hours after the procedure, and chart review looking for other events, as is the standard at our institution.

\section{Statistical methods}

Descriptive statistics are presented for all demographic data including age, gender, race and reason for EUS-FNA. Continuous variables are reported as median and inter-quartile range, and categorical variables are shown as frequency and percentage. Comparisons between the cases (heparin priming) and controls (no heparin priming) were made using Wilcoxon Ranksum and Pearson's Chi-square tests, as appropriate. Data analysis was performed using Systat version 13 (Systat Software, San Jose, CA), and SPSS version 22 (IBM Corp., Armonk, NY). A $P<0.05$ was considered statistically significant.

\section{Results}

Data from a total of 73 EUS-FNA procedures was evaluated during our study period. Of the EUS-FNA procedures, 37 were performed with heparin and 36 without heparin. Of the EUS-FNAs, 31 (84\%) were performed with the dry heparin technique, and $6(16 \%)$ using the wet heparin technique. 
- Table 1 Demographics.

\begin{tabular}{|c|c|c|c|c|}
\hline & & $\begin{array}{l}\text { Heparin } \\
\mathbf{n}=37\end{array}$ & $\begin{array}{l}\text { No heparin } \\
n=36\end{array}$ & $P$ value \\
\hline Mean age (years) (IQR) & & $71(63.5-77.5)$ & $74(66.5-80.5)$ & $0.83^{1}$ \\
\hline \multirow[t]{2}{*}{ Sex } & Female & 20 & 18 & 0.91 \\
\hline & Male & 17 & 18 & \\
\hline \multirow[t]{3}{*}{ Race } & Caucasian & 33 & 35 & 0.79 \\
\hline & African american & 2 & 1 & \\
\hline & Southeast asian & 2 & 0 & \\
\hline \multirow[t]{8}{*}{ Location } & Pancreatic & 12 & 13 & 0.99 \\
\hline & Lymph node & 12 & 10 & \\
\hline & Liver & 7 & 6 & \\
\hline & Left adrenal & 2 & 3 & \\
\hline & Gastric & 2 & 2 & \\
\hline & Mediastinal mass & 1 & 1 & \\
\hline & Peritoneal mass & 0 & 1 & \\
\hline & Thyroid mass & 1 & 0 & \\
\hline \multirow[t]{6}{*}{ EUS } & Median needle passes (IQR) & $4(3-6)$ & $4(3-6)$ & 0.74 \\
\hline & $22 \mathrm{~g}$ core & 6 & 2 & 0.39 \\
\hline & $22 \mathrm{~g}$ & 1 & 4 & \\
\hline & $25 \mathrm{~g}$ & 30 & 30 & \\
\hline & Dry heparin & 29 & 0 & \\
\hline & Wet heparin & 6 & 0 & \\
\hline
\end{tabular}

Among all cases, $46 \%$ were male and $95 \%$ were Caucasian. There were no significant differences in the study demographics ( $\triangleright$ Table 1 ). A median of 4 needle passes (interquartile range $3-6)$ were made for every discrete lesion sampled with heparin and non-heparin EUS-FNA $(P=0.74)$.

No procedure-related adverse events and specifically no episodes of bleeding after FNA were observed. There were no admissions for medical care required in any subjects after their procedure.

\section{FNA indications}

A total of 37 lesions were sampled with heparin priming, among which pancreatic masses (12) were the most common FNA target in the heparin group (32\%). This was followed by followed by lymph nodes 12 (32\%), liver masses 7 (19\%), left adrenal masses 2 (5\%), and gastric lesions 2 (5\%). There was a single mediastinal mass (3\%), one thyroid mass in the heparin group (3\%), and one peritoneal mass in the non-heparin group (3\%).

\section{FNA histopathologic characteristics}

A final diagnosis of malignancy was made in $87 \%$ of heparin cases and $86 \%$ of non-heparin cases. A summary of all clinical diagnoses by location is depicted in $\mathbf{r}$ Table 2 . Of all proven malignant lesions, 31 (100\%) were positive for malignancy in the heparin group and $94 \%$ in the non-heparin group, none were read as "suspicious" or atypical. Two lesions were carcinoma of unknown primary; one was a poorly differentiated carcinoma and a second an "unclassified malignancy." There was 1 case of scant cellularity in the heparin group and two in the control group. In non-malignant lesions, a diagnosis was made in 6 and 5 , respectively (heparin v. non-heparin), and malignancy ruled out in these patients (100\%). Details are provided in > Table 2.

IHC staining was done in 28 heparinized cases and 32 nonheparinized cases. Staining using IHC was successful in all but one $(96 \%)$ of cases in the heparin group, and $94 \%$ in the nonheparin group $(P=0.53)$. All 3 failures of IHC were due to lack of cellularity of the specimen. A wide variety of IHC targets were used (48 different markers), and heparin priming of the 
- Table 2 Histopathologic characteristics of EUS FNA procedures.

\begin{tabular}{|c|c|c|c|c|}
\hline & & $\begin{array}{l}\text { Heparin } \\
\mathbf{n}=37\end{array}$ & $\begin{array}{l}\text { No heparin } \\
\mathrm{n}=36\end{array}$ & $P$ value $^{1}$ \\
\hline \multirow[t]{3}{*}{ Flow cytometry result } & Benign & 3 & 4 & 1 \\
\hline & B-cell lymphoma & 2 & 1 & \\
\hline & & & & 0.53 \\
\hline \multirow[t]{3}{*}{ IHC result } & Complete & 27 & 30 & \\
\hline & Not possible & 1 & 2 & \\
\hline & Not done & 9 & 4 & \\
\hline FNA site & Diagnosis & & & \\
\hline \multirow[t]{4}{*}{ Pancreatic mass } & Adeno pancreas & 9 & 11 & 0.96 \\
\hline & Lymphoma & 1 & 0 & \\
\hline & Mucinous & 1 & 1 & \\
\hline & NET & 1 & 1 & \\
\hline \multirow[t]{4}{*}{ Liver mass } & Adeno pancreas & 2 & 4 & \\
\hline & Adeno colon & 2 & 0 & \\
\hline & Biliary mucinous neoplasm & 2 & 0 & \\
\hline & NSC & 1 & 1 & \\
\hline \multirow[t]{7}{*}{ Lymph node } & Adeno pancreas & 1 & 1 & \\
\hline & Adeno breast & 0 & 1 & \\
\hline & $\mathrm{HCC}$ & 1 & 0 & \\
\hline & Lymphoma & 2 & 1 & \\
\hline & NET & 1 & 2 & \\
\hline & NSC & 1 & 1 & \\
\hline & Benign & 6 & 4 & \\
\hline \multirow[t]{4}{*}{ Adrenal } & Adeno pancreas & 1 & 0 & \\
\hline & Adeno colon & 0 & 2 & \\
\hline & Adeno lung & 0 & 1 & \\
\hline & RCC & 1 & 0 & \\
\hline \multirow[t]{3}{*}{ Gastric } & Adeno & 0 & 1 & \\
\hline & GIST & 1 & 1 & \\
\hline & Leiomyoma & 1 & 0 & \\
\hline Mediastinal mass & NSC & 1 & 1 & \\
\hline Peritoneal mass & Carcinoma & 0 & 1 & \\
\hline Thyroid & Malignant & 1 & 0 & \\
\hline
\end{tabular}

needle did not alter expression and/or detection of these markers.

Additionally, flow cytometry was performed in 5 cases in each group, $100 \%$ of which were successful in making a clinical diagnosis. Of such cases, 3 v. 4 (heparin and non-heparin respectively) cases were benign lymph nodes and 2 v. 1 were found to have B-cell lymphoma. 


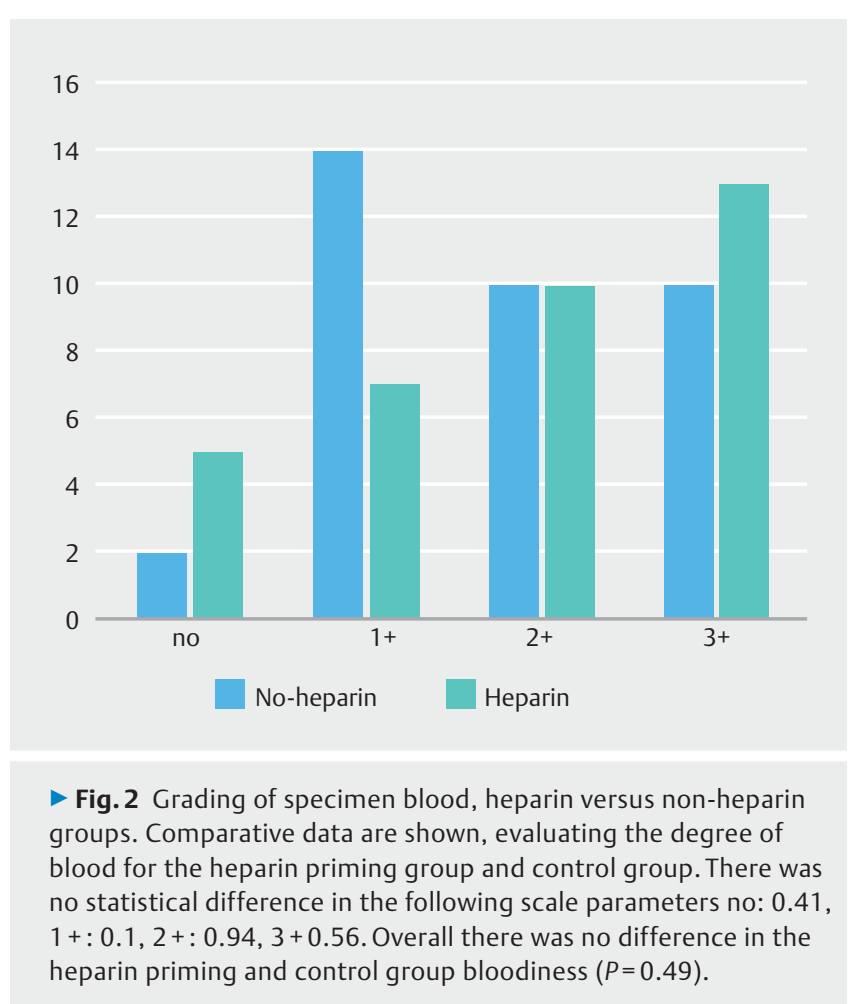

Using the objective scale to quantify the degree of specimen bloodiness, there was no overall difference between the heparin versus non-heparin groups. Additionally, there was no statistical difference in any of the 4 grades of bloodiness in the heparin versus non-heparin group ( $\triangleright$ Fig. 2 ).

\section{Discussion}

This study is the first to show that use of a heparin-primed EUSFNA needle does not affect cytological or histological interpretation, nor expression and/or detection of a variety of IHC markers. In addition, specimens were not bloodier than those obtained without use of heparin.

The first published report of the use of heparin to prime the FNA needle was from a 1985 study of percutaneous FNA of liver lesions (hepatomas) [9]. Heparin priming was found to increase both diagnostic and tissue yields. This study also found that additional small tissue fragments could be collected which would be otherwise trapped in the needle, and therefore also increased the diagnostic yield.

The wet suction technique ("WEST") has been shown to increase EUS-FNA specimen yields [7]. In this study, the investigators primed the needle with saline prior to performing FNA, and compared this technique to conventional EUS-FNA. Wet suction using heparin instead of saline ("wet heparin" technique) led to no significant difference in FNA specimen cellularity, adequacy, or degree of blood contamination. The results of the current study suggest that heparin priming can be safely used for the WEST technique, rather than saline.

A technical "trick" to manage blood clogging of the EUS needle is to flush the needle with heparin [11]. Conversely, a text- book of EUS states that the introduction of heparin into cytologic specimens could negatively affect the cytologic yield [12]. A more recent study sought to determine if heparin priming of the EUS-FNA needle led to improved diagnostic yields [10]. However, no difference in diagnostic accuracy, number of needle passes or blood contamination for heparin as compared with non-heparin priming technique was found.

In this study, we have found that heparin priming does not lead to degradation of specimens, performance of IHC staining, or difficulty in diagnostic interpretation. The "dry heparin" technique in particular uses only enough heparin to coat the inside of the needle. Even this small amount of anticoagulation appears to largely prevent blood clotting within the needle. An evaluation of cellblock bloodiness in our study found no difference in amount of blood with heparin compared to a historical control group of FNA done without use of heparin.

Gastroenterology technicians and nurses who work with EUS needles on a regular basis can attest to the progressive difficulty with stylet reinsertion in subsequent passes. This may be particularly difficult with the 25 -g needle, whose stylet is correspondingly small, and difficult to manipulate. We have also found that heparin priming of the needle, even if only at the beginning of sampling ("dry heparin") can lead to easier stylet passage into the needle, even up to 7 stylet insertions. Thus, heparin priming of the needle could potentially be done in every case to simplify the job of the endoscopy assistant in handling the specimen and the stylet.

There may be concern for an increased risk of bleeding after FNA with a heparin-primed needle. However, we did not see any episodes of bleeding with either the dry or wet heparin technique. The amount of heparin is small, the area of tissue exposed to heparin on the needle tip tiny, and heparin in the needle is being suctioned away from the target lesion, and not injected into it.

Potential weaknesses of this study included the retrospective analysis of a prospectively recorded database, which does not possess the scientific rigor of a prospective trial. Target and needle size heterogeneity may have also be a source of study weakness, however despite this variability we did not find a difference in several cytologic outcomes. A strength of this study was a successful proof of concept in a variety of situations that are relevant to the endosonographer anatomically, histologically and clinically.

\section{Conclusion}

In conclusion, we strongly favor heparin priming of the EUS needle prior to use in all procedures utilizing FNA. Even if the flush is done only once ("dry heparin" technique), subsequent prevention of blood clot formation within the needle lumen is observed, which can potentially make expression of tissue from the needle much easier. Heparin can be used instead of saline if the "wet suction" technique is being used. Heparin priming of the needle appears to be safe. There were no deleterious effects on cytological or histological interpretation, and IHC staining is preserved over a wide range of stains. 


\section{Acknowledgements}

The authors of this study would like to thank Dustin Lin and Alicia Kovach for assisting with data extraction.

Competing interests

Dr. Khara is a consultant for Medtronic-Covidien. Dr. Johal is a consultant for Boston Scientific Corporation. Dr. Diehl is a consultant for Boston Scientific Corporation and Olympus.

\section{References}

[1] Dumonceau JM, Polkowski M, Larghi A et al. Indications, results, and clinical impact of endoscopic ultrasound (EUS)-guided sampling in gastroenterology. European Society of Gastrointest Endosc 2011; 43: 897-909

[2] Sahai AV, Paquin SC, Gariépy G. A prospective comparison of endoscopic ultrasound-guided fine needle aspiration results obtained in the same lesion, with and without the needle stylet. Endoscopy 2010; 42: $900-903$

[3] Rastogi A, Wani S, Gupta N et al. A prospective, single-blind, randomized, controlled trial of EUS-guided FNA with and without a stylet. Gastrointest Endosc 2011; 74: 58-64

[4] Puri R, Vilmann P, Săftoiu A et al. Randomized controlled trial of endoscopic ultrasound-guided fine-needle sampling with or without suction for better cytological diagnosis. Scand ] Gastroenterol 2009; 44: $499-504$

[5] Bang JY, Magee SH, Ramesh J et al. Randomized trial comparing fanning with standard technique for endoscopic ultrasound-guided fineneedle aspiration of solid pancreatic mass lesions. Endoscopy 2013; 45: $445-450$

[6] Nakai $\mathrm{Y}$, Isayama $\mathrm{H}$, Chang $\mathrm{KJ}$ et al. Slow pull versus suction in endoscopic ultrasound-guided fine-needle aspiration of pancreatic solid masses. Dig Dis Sci 2014; 59: 1578 - 1585

[7] Attam R, Arain MA, Bloechl SJ et al. "Wet suction technique (WEST)": a novel way to enhance the quality of EUS-FNA aspirate. Results of a prospective, single-blind, randomized, controlled trial using a 22gauge needle for EUS-FNA of solid lesions. Gastrointest Endosc 2015; 81: $1401-1407$

[8] Varadarajulu S, Hasan MK, Bang JY et al. Endoscopic ultrasound-guided tissue acquisition. Dig Endosc 2014; 26: 62 - 69

[9] Kasugai H, Yamamoto R, Tatsuta $M$ et al. Value of heparinized fineneedle aspiration biopsy in liver malignancy. Am J Roentgenol 1985; 144: $243-244$

[10] Hasan MK, Bang JY, Varadarajulu S. Diagnostic value of priming the endoscopic ultrasound-guided fine-needle aspiration needle with heparin to improve specimen quality. Digestive Endosc 2014; 26: 491

[11] Puri R, Thandassery RB, Choudhary NS et al. Endoscopic ultrasoundguided fine-needle aspiration of the adrenal glands: analysis of 21 patients. Clin Endosc 2015; 48: 165 - 170

[12] Hawes RH, Fockens P, Varadarajulu S. Endosonography, ${ }^{\text {3rd Ed. St. }}$ Louis, MO: Elsevier Saunders; 2014 